\title{
First-order spatial coherence of excitons in planar nanostructures: A $k$-filtering effect
}

\author{
L. Mouchliadis and A. L. Ivanov \\ Department of Physics and Astronomy, Cardiff University, Queens Buildings, CF24 3AA Cardiff, United Kingdom
}

(Received 16 January 2008; revised manuscript received 15 May 2008; published 22 July 2008)

\begin{abstract}
We propose and analyze a $k_{\|}$-filtering effect which gives rise to the drastic difference between the actual spatial coherence length of quasi-two-dimensional excitons or microcavity polaritons in planar nanostructures and that inferred from far-field optical measurements. The effect originates from conservation of the in-plane wave-vector $k_{\|}$in the optical decay of the particles in outgoing bulk photons. The $k_{\|}$-filtering effect explains the large coherence lengths recently observed for indirect excitons in coupled quantum wells but is less pronounced for microcavity polaritons at low temperatures, $T \lesssim 10 \mathrm{~K}$.
\end{abstract}

DOI: 10.1103/PhysRevB.78.033306

PACS number(s): 78.67.De, 71.35.-y, 42.50.Ar

Long-range spatial coherence is a fingerprint of welldeveloped Bose-Einstein (BE) statistics. Measurements of the first-order spatial coherence function $g^{(1)}$ and the coherence length $\xi$ have allowed visualization of the BE condensation transition in a trapped Bose gas of $\mathrm{Rb}$ atoms. ${ }^{1}$ There are several recent reports on the observation of long-range spatial optical coherence in a low-temperature quasi-twodimensional (quasi-2D) system of microcavity (MC) polaritons ${ }^{2,3}$ and indirect excitons. ${ }^{4-7}$ In this case, the resonant optical decay of MC polaritons or quantum well (QW) excitons in bulk photon modes allows mapping of the inplane coherence function $g^{(1)}$ of the particles by measuring the optical coherence function $\widetilde{g}^{(1)}$ of the emitted photons. It is commonly assumed that the coherence length of QW excitons (MC polaritons), $\xi_{x}\left(\xi_{p}\right)$, associated with $g^{(1)}$, is identical to that, $\xi_{\gamma}$, of the optical coherence function $\tilde{g}^{(1)}$.

In this Brief Report, we report a $k_{\|}$-filtering effect, which can strongly influence the optical coherence function $\widetilde{g}^{(1)}$ measured from a planar nanostructure, and calculate $g^{(1)}$ and $\widetilde{g}^{(1)}$ for QW excitons and MC polaritons. For QW excitons, the $k_{\|}$-filtering effect tremendously increases the optical coherence length $\xi_{\gamma}$, leading to $\xi_{\gamma} \gg \xi_{x}$, and can naturally explain the micron coherence lengths observed for indirect excitons and attributed to spontaneously developed coherence. The effect is less pronounced for MC polaritons, still with $\xi_{\gamma} \geq \xi_{p}$.

The $k_{\|}$-filtering effect stems from the energy and in-plane momentum conservation in the resonant conversion "quasi-2D QW exciton (MC polariton) $\rightarrow$ outgoing bulk photon." For a QW structure surrounded by thick coplanar barrier layers, the case illustrated in Fig. 1, only low-energy optically active excitons from the radiative zone $k_{\|} \leq k_{0}$ $=\left(\sqrt{\varepsilon_{b}} / c\right) \omega_{0}$, with $\varepsilon_{b}$ the dielectric constant of barrier layers and $\hbar \omega_{0}$ the exciton energy at $k_{\|}=0$, are bright, i.e., can emit far-field light. ${ }^{8-11}$ In a far-field optical experiment with detection angle $2 \alpha$ [see Fig. 1(b)], the fraction of QW excitons which contribute to the optical signal is drastically further reduced to the wave-vector band $\Delta k_{\|}$given by $0 \leq k_{\|} \leq k_{\|}^{(\alpha)}$ $=\left(k_{0} / \sqrt{\varepsilon_{b}}\right) \sin \alpha \ll k_{0}$. The $\alpha$-dependent narrowing of the detected states results in an effective broadening of the firstorder spatial coherence function $\widetilde{g}^{(1)}$. In addition, the sharp cutoff of the detected states at $k_{\|}=k_{\|}^{(\alpha)}$ yields an unusual oscillatory behavior of $\widetilde{g}^{(1)}$. The $k_{\|}$-filtering effect has no analogy in optics of bulk excitons or polaritons.
The first-order spatial coherence function $g^{(1)}$ (Refs. 12 and 13) of quantum well excitons, at a fixed time, is given by $g^{(1)}\left(\mathbf{r}_{\|}^{\prime}, \mathbf{r}_{\|}^{\prime \prime}\right)=G^{(1)}\left(\mathbf{r}_{\|}^{\prime}, \mathbf{r}_{\|}^{\prime \prime}\right) /\left[G^{(1)}\left(\mathbf{r}_{\|}^{\prime}, \mathbf{r}_{\|}^{\prime}\right) G^{(1)}\left(\mathbf{r}_{\|}^{\prime \prime}, \mathbf{r}_{\|}^{\prime \prime}\right)\right]^{1 / 2}$, with $G^{(1)}\left(\mathbf{r}_{\|}^{\prime}, \mathbf{r}_{\|}^{\prime \prime}\right)=\left\langle\hat{\Psi}^{\dagger}\left(\mathbf{r}_{\|}^{\prime}\right) \hat{\Psi}\left(\mathbf{r}_{\|}^{\prime \prime}\right)\right\rangle, \quad$ where $\hat{\Psi}\left(\mathbf{r}_{\|}^{\prime}\right)$ $=(1 / \sqrt{S}) \Sigma_{\mathbf{k}_{\|}} e^{i \mathbf{k}_{\|} \mathbf{r}_{\|}^{\prime}} B_{\mathbf{k}_{\|}}, \mathbf{r}_{\|}^{\prime}$ is the in-plane coordinate, $S$ is the area, and $B_{\mathbf{k}_{\|}}$is the exciton operator. Thus for isotropically distributed $\mathrm{QW}$ excitons one receives

$$
g^{(1)}=g^{(1)}\left(r_{\|}\right)=\frac{1}{2 \pi n_{2 d}} \int_{0}^{\infty} J_{0}\left(k_{\|} r_{\|}\right) n_{k_{\|}} k_{\|} d k_{\|},
$$

where $r_{\|}=\left|\mathbf{r}_{\|}^{\prime \prime}-\mathbf{r}_{\|}^{\prime}\right|, n_{2 d}$ is the concentration of particles, $n_{\mathbf{k}_{\|}}$ $=\left\langle B_{\mathbf{k}_{\|}}^{\dagger} B_{\mathbf{k}_{\mathbf{k}}}\right\rangle$ is the occupation number, and $J_{0}$ is the zerothorder Bessel function of the first kind. For a classical gas of QW excitons at thermal equilibrium, Eq. (1), with $n_{\mathbf{k}_{\|}}$given by the Maxwell-Boltzmann (MB) distribution function $n_{k_{\|}}^{\mathrm{MB}}$, yields the well-known result, ${ }^{3,14}$ (a)

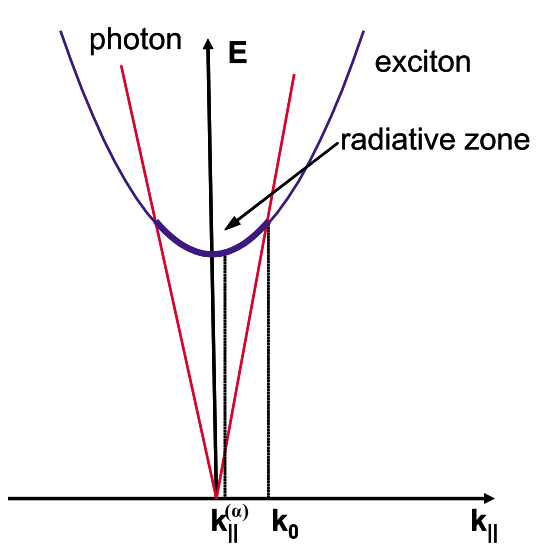

(b)

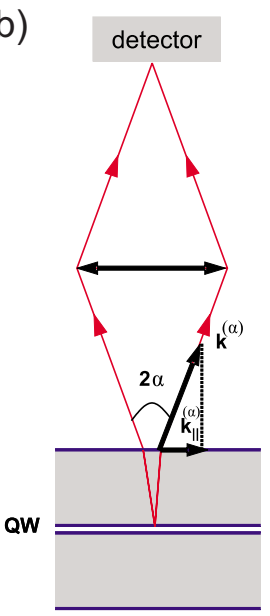

FIG. 1. (Color online) Schematic of the $k_{\|}$filtering effect. (a) The exciton and photon dispersions. Only low-energy QW excitons from the radiative zone $k_{\|} \leq k_{0}$ can emit outgoing bulk photons. (b) A far-field optical experiment with detection angle $2 \alpha$ : a small fraction of QW excitons with $\left|\mathbf{k}_{\|}\right| \leq k_{\|}^{(\alpha)}=\left(k_{0} / \sqrt{\varepsilon_{b}}\right) \sin \alpha$ contributes to the optical signal. 


$$
g^{(1)}=g_{\mathrm{cl}}^{(1)}\left(r_{\|}\right)=e^{-\pi r_{\|}^{2} / \lambda_{\mathrm{dB}}^{2}},
$$

where the thermal de Broglie wavelength is given by $\lambda_{\mathrm{dB}}$ $=\left[\left(2 \pi \hbar^{2}\right) /\left(M_{x} k_{B} T\right)\right]^{1 / 2}$, with $T$ the temperature and $M_{x}$ the exciton in-plane translational mass. For helium temperatures, one estimates from Eq. (2) the coherence length of MBdistributed indirect excitons in GaAs coupled QWs as $\xi_{x}$ $\sim \lambda_{\mathrm{dB}} \sim 0.1 \mu \mathrm{m}$.

Compared with Eq. (1), the spatial coherence function $\widetilde{g}^{(1)}$ of photons emitted by QW excitons is given by

$$
\widetilde{g}^{(1)}\left(r_{\|}\right)=\frac{\int_{0}^{\infty} G_{f}\left(k_{\|}\right) J_{0}\left(k_{\|} r_{\|}\right) n_{k_{\|}} k_{\|} d k_{\|}}{\int_{0}^{\infty} G_{f}\left(k_{\|}\right) n_{k_{\|}} k_{\|} d k_{\|}},
$$

where $G_{f}=\Theta\left(k_{\|}^{(\alpha)}-k_{\|}\right) \Gamma_{x-\gamma}\left(k_{\|}\right)$is the $k_{\|}$-filtering function, with $\Theta(x)$ the step function and $\Gamma_{x-\gamma}\left(k_{\|}\right)$the efficiency of the resonant conversion of a QW exciton in an outgoing bulk photon. The function $G_{f}$ reduces the integration limits on the right-hand side (rhs) of Eq. (3) to the narrow band $\Delta k_{\|}$ $=\left[0, k_{\|}^{(\alpha)}\right]$ and describes the $k_{\|}$-filtering effect in high-quality planar nanostructures. If both the function $\Gamma_{x-\gamma}\left(k_{\|}\right)$and the occupation number $n_{k_{\|}}$do not change significantly in the narrow band $\Delta k_{\|}$, Eq. (3) yields

$$
\widetilde{g}^{(1)}=\widetilde{g}_{f}^{(1)}\left(r_{\|}\right)=2 J_{1}\left(k_{\|}^{(\alpha)} r_{\|}\right) /\left(k_{\|}^{(\alpha)} r_{\|}\right),
$$

where $J_{1}$ is the first-order Bessel function of the first kind. From Eq. (4) one concludes that the optical coherence length $\xi_{\gamma}$, evaluated as the half width at half maximum of $\widetilde{g}^{(1)}$ $=\widetilde{g}_{f}^{(1)}\left(r_{\|}\right)$, is given by

$$
4 J_{1}\left(k_{\|}^{(\alpha)} \xi_{\gamma}\right)=k_{\|}^{(\alpha)} \xi_{\gamma} \rightarrow k_{\|}^{(\alpha)} \xi_{\gamma} \simeq 2.215 .
$$

Equations (4) and (5) illustrate the net $k_{\|}$-filtering effect in the absence of instrumental aberrations: $\xi_{\gamma} \propto 1 / k_{\|}^{(\alpha)}$ $\propto 1 / \sin \alpha$ strongly increases with decreasing aperture angle $2 \alpha$. Below we analyze in more detail the exciton function $g^{(1)}$ against the optical $\widetilde{g}^{(1)}$, assuming no phase transition to a collective (superfluid) state.

First-order spatial coherence of noninteracting quasi-2D bosons (excitons) in equilibrium. In this case, the chemical potential $\mu_{2 d}$ is given by $\mu_{2 d}^{(0)}=k_{B} T \ln \left(1-e^{-T_{0} / T}\right)$, with $k_{B} T_{0}$ $=(2 \pi / g)\left(\hbar^{2} / M_{x}\right) n_{2 d}$ the quantum degeneracy temperature where $g$ is the spin degeneracy factor of bosons $(g=4$ for indirect excitons). By substituting $n_{k_{\|}}=n_{k_{\|}}^{\mathrm{BE}}$ into Eq. (1), where $n_{k_{\|}}^{\mathrm{BE}}$ is the Bose-Einstein occupation number, one receives

$$
\begin{aligned}
g^{(1)} & =g_{\text {nint }}^{(1)}\left(r_{\|}\right)=\frac{T}{T_{0}} g_{1}\left(1-e^{T_{0} / T}, e^{-\pi r_{\|}^{2} / \lambda_{\mathrm{dB}}^{2}}\right) \\
& =\frac{T}{T_{0}} \sum_{n=1}^{\infty} \frac{\left(1-e^{-T_{0} / T}\right)^{n}}{n} e^{-\pi r_{\|}^{2} / n \lambda_{\mathrm{dB}}^{2}}
\end{aligned}
$$

Here, the generalized Bose function ${ }^{14} g_{\nu}(x, y)$ with $\nu=1$ is defined as $g_{\nu}(x, y)=\sum_{k=1}^{\infty}\left(x^{k} y^{1 / k}\right) / k^{\nu}$.
For distances $r_{\|} \gtrsim r_{\|}^{(q)}=\lambda_{\mathrm{dB}}\left[-(2 / \pi) \ln \left(1-e^{-T_{0} / T}\right)\right]^{1 / 2} \mathrm{Eq}$. (6) reduces to the quantum limit when the sum on the rhs cannot be approximated by the first term,

$$
g^{(1)}\left(r_{\|} \gtrsim r_{\|}^{(q)}\right) \simeq 2 \frac{T}{T_{0}} K_{0}\left(\frac{r_{\|}}{r_{0}}\right),
$$

where $K_{0}$ is the modified Bessel function of the second kind and $r_{0}=\lambda_{\mathrm{dB}} /\left[-4 \pi \ln \left(1-e^{-T_{0} / T}\right)\right]^{1 / 2}$. For $r_{\|} \gtrsim r_{0} \gtrsim r_{\|}^{(q)}$, Eq. (7) reduces further to

$$
g^{(1)}=g_{q}^{(1)}\left(r_{\|} \gtrsim r_{0}\right)=\sqrt{2 \pi} \frac{T}{T_{0}} \sqrt{\frac{r_{0}}{r_{\|}}} e^{-r_{\|} / r_{0}} .
$$

For temperatures $T \gg T_{0}$, the spatial coherence function is well approximated by Eq. (2), and the quantum corrections given by Eqs. (7) and (8) refer to large $r_{\|} \gtrsim r_{\|}^{(q)}$ $\simeq \lambda_{\mathrm{dB}} \sqrt{(2 / \pi) \ln \left(T / T_{0}\right)} \gg \lambda_{\mathrm{dB}}$ and, therefore, to very small values of $g^{(1)}$. For $T \leqq T_{0}$, when BE statistics is well developed, Eqs. (7) and (8) are valid for distances larger than $r_{\|}^{(\mathrm{q})}$ $\simeq \lambda_{\mathrm{dB}} \sqrt{(2 / \pi)} e^{-T_{0} / 2 T} \ll \lambda_{\mathrm{dB}}$, so that $g^{(1)}$ is well approximated by $g_{q}^{(1)}$ for any $r_{\|}$. The quantum statistical effects, which are included in Eq. (7) through $T_{0} \propto \hbar^{2}$, considerably increase the correlation length $\xi_{x}$, giving rise to $\xi_{x} \simeq\left[\lambda_{\mathrm{dB}} /(2 \sqrt{\pi})\right] e^{T_{0} / 2 T}$ for $T \lesssim T_{0}$ (see Fig. 2).

The coherence function $g^{(1)}$ of weakly interacting thermal $Q W$ excitons. For circularly polarized excitons in single QWs, the case relevant to MC polaritons, the repulsive interaction between the particles is well approximated by a contact potential $U_{\mathrm{SQW}}=\left(u_{0} / 2\right) \delta\left(\mathbf{r}_{\|}\right)$, with $u_{0}=u_{0}^{\mathrm{SQW}}>0$. In this case, the mean-field Hartree-Fock (HF) interaction only shifts the chemical potential, $\mu_{2 d}=\tilde{\mu}_{2 d}^{(0)}=\mu_{2 d}^{(0)}+u_{0} n_{2 d}$, leaving unchanged Eqs. (6)-(8).

For indirect excitons in coupled QWs, the mid-range dipole-dipole repulsive interaction $U_{\mathrm{CQW}}$ of the particles cannot be generally approximated by a contact potential. Following Ref. 15, we use the two-parametric model potential $U_{\mathrm{CQW}}\left(r_{\|}\right)=\left[\left(\sqrt{\pi} u_{0} w\right) / r_{\|}^{3}\right]\left(1-e^{-r_{\|}^{2} / w^{2}}\right)$, with parameters $u_{0}$ $=u_{0}^{\mathrm{CQW}} \simeq 4 \pi\left(e^{2} / \varepsilon_{b}\right) d_{z}$ (Refs. 16 and 17) and $w \simeq a_{x}^{(2 d)}$, where $d_{z}$ is the distance between coupled quantum wells and $a_{x}^{(2 d)}$ is the radius of an indirect exciton. The model potential reproduces $1 / r_{\|}^{3}$ behavior at $r_{\|} \gtrsim a_{x}^{(2 d)}$ and $1 / r_{\|}$Coulomb repulsive potential at $r_{\|} \lesssim a_{x}^{(2 d)}$. The self-consistent HF analysis ${ }^{18}$ of the Hamiltonian $H_{x}=\Sigma_{\mathbf{p}_{\|}}\left[p_{\|}^{2} /\left(2 M_{x}\right)\right] B_{\mathbf{p}_{\|}^{\dagger}}^{\dagger} B_{\mathbf{p}_{\|}}+1 /(2 S) \Sigma_{\mathbf{p}_{\|}, \mathbf{l}_{\|}, \mathbf{q}_{\|}}$ $U_{\mathrm{CQW}}\left(\mathbf{q}_{\|}\right) B_{\mathbf{p}_{\|}}^{\dagger} B_{\mathbf{1}_{\|}}^{\dagger} B_{\mathbf{1}_{\|}+\mathbf{q}_{\|}} B_{\mathbf{p}_{\|}-\mathbf{q}_{\|}}$yields the $n_{2 d^{-}}$and $T$-dependent change in the in-plane translational mass $M_{x}$. In this case, $\mu_{2 d}$ is

$$
\begin{aligned}
\mu_{2 d}= & \tilde{\mu}_{2 d}^{(0)}+\frac{u_{0}}{2\left(\lambda_{\mathrm{dB}}^{*}\right)^{2}}\left\{\frac{T_{0}^{*}}{T}+\sqrt{\pi} \frac{w}{\lambda_{\mathrm{dB}}^{*}}\left[\frac{\sqrt{\pi}}{2} \frac{w}{\lambda_{\mathrm{dB}}^{*}} \operatorname{Li}_{2}(F)\right.\right. \\
& \left.\left.-\mathrm{Li}_{3 / 2}(F)\right]\right\},
\end{aligned}
$$

where, together with Eq. (6), both the de Broglie wavelength $\lambda_{\mathrm{dB}}^{*}$ and the degeneracy temperature $T_{0}^{*}$ are changed according to $M_{x} \rightarrow M_{x}^{*}, F=1-e^{-T_{0}^{*} / T}$, and $\operatorname{Li}_{\nu}(x)=\sum_{k=1}^{\infty} x^{k} / k^{\nu}$ is the polylogarithm. The particle mass $M_{x}^{*}$ renormalized by the dipole-dipole interaction is given as a single solution of the transcendental equation, 

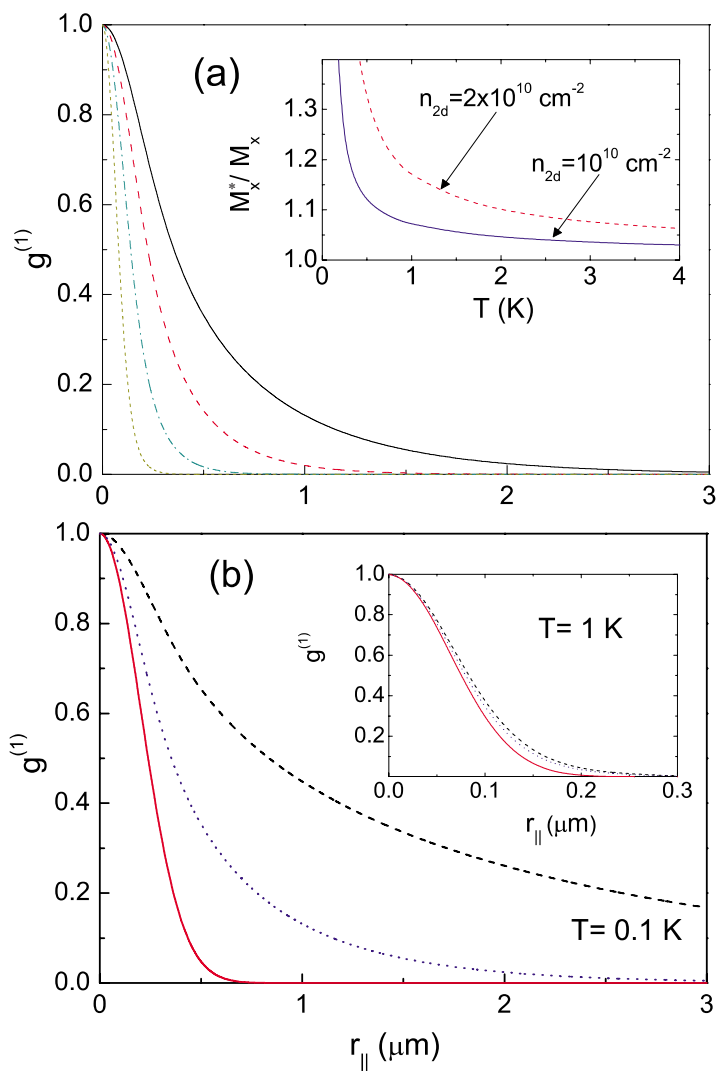

FIG. 2. (Color online) (a) The first-order spatial coherence function $g^{(1)}=g_{\text {ind }}^{(1)}\left(r_{\|}\right)$of indirect excitons in a GaAs coupled QW structure with $d_{z}=11.5 \mathrm{~nm}$ and $w=15 \mathrm{~nm}: n_{2 d}=10^{10} \mathrm{~cm}^{-2}$ and $T=1$ (dotted line), 0.4 (dash-dotted line), 0.2 (dashed line), and $0.1 \mathrm{~K}$ (solid line). Inset: the renormalized mass $M_{x}^{*}$ against temperature $T$, calculated with Eq. (10). (b) $g^{(1)}=g_{\mathrm{cl}}^{(1)}\left(r_{\|}\right)$(solid line), $g^{(1)}$ $=g_{\text {nint }}^{(1)}\left(r_{\|}\right) \quad$ (dashed line), and $g^{(1)}=g_{\text {ind }}^{(1)}\left(r_{\|}\right) \quad\left(\right.$ dotted line): $n_{2 d}$ $=10^{10} \mathrm{~cm}^{-2}$ and $T=0.1 \mathrm{~K}$. Inset: the same functions evaluated for $n_{2 d}=10^{10} \mathrm{~cm}^{-2}$ and $T=1 \mathrm{~K}$.

$$
\frac{1}{M_{x}^{*}}=\frac{1}{M_{x}}+\frac{u_{0} w}{8 \sqrt{\pi} \hbar^{2} \lambda_{\mathrm{dB}}^{*}}\left[\sqrt{\pi} \frac{w}{\lambda_{\mathrm{dB}}^{*}} \frac{T_{0}^{*}}{T}-\mathrm{Li}_{1 / 2}(F)\right] .
$$

In Fig. 2(a) we plot $g^{(1)}=g_{\text {ind }}^{(1)}\left(r_{\|}\right)$evaluated numerically by using Eqs. (6), (9), and (10) for indirect excitons in a GaAs coupled QW structure. In Fig. 2(b), the coherence function $g_{\text {ind }}^{(1)}$ is compared with $g_{\mathrm{cl}}^{(1)}$ evaluated with Eq. (2) and $g_{\text {nint }}^{(1)}$ calculated with Eq. (6) for noninteracting excitons. The main result is that the dipole-dipole repulsive interaction induces an increase in the translational mass [see the inset of Fig. $2(\mathrm{a})]$ and, therefore, decreases the coherence length $\xi_{x}$ compared to that of noninteracting particles [see also Fig. 3(a)]. The effect, however, becomes pronounced only at temperatures well below $1 \mathrm{~K}$. For $T=1 \mathrm{~K}$ all three correlation functions, $g_{\text {ind }}^{(1)}, g_{\text {cl }}^{(1)}$, and $g_{\text {nint }}^{(1)}$, nearly coincide, as is clearly seen in the inset of Fig. 2(b). In other words, for $n_{2 d}=10^{10} \mathrm{~cm}^{-2}$ and $T=1.5 \mathrm{~K}$, which are relevant to the experiments, ${ }^{4-7}$ the quantum limit, i.e., $g^{(1)}=g_{q}^{(1)}$ given by Eq. (8), cannot build up: One estimates $T_{0} \simeq T_{0}^{*} \approx 0.65 \mathrm{~K}$ and $n_{k_{\|}=0}^{\mathrm{BE}} \approx 0.54<1$, so that $\mathrm{BE}$ statistics is rather weakly developed to influence the coherence length $\xi_{x}$.

The given description of $g^{(1)}$ refers to temperatures above
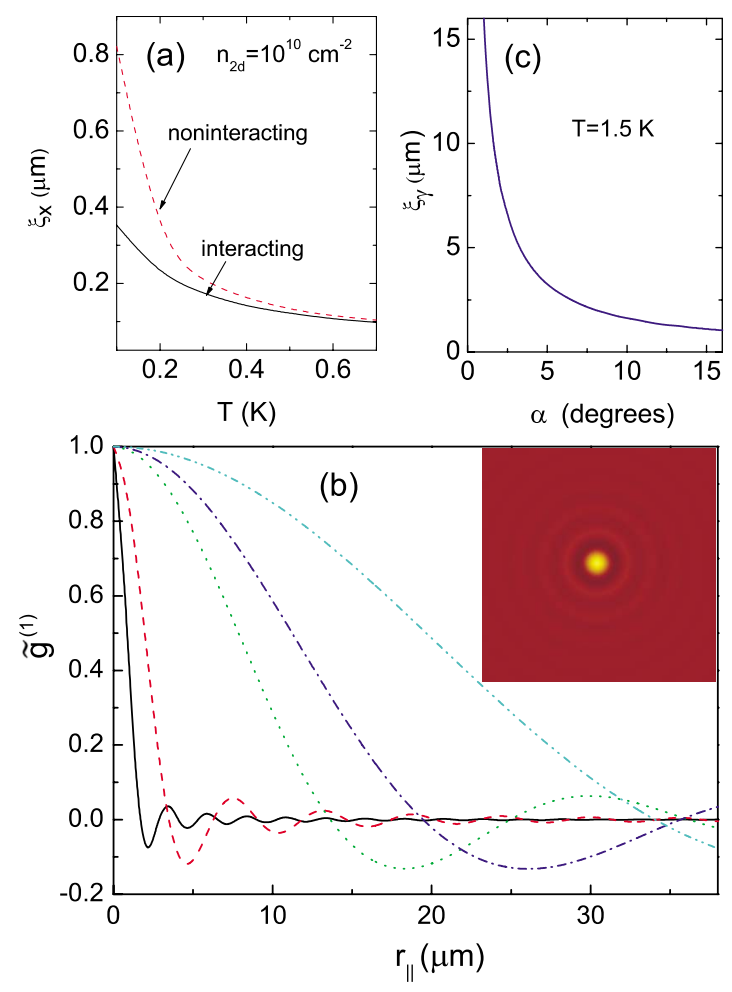

FIG. 3. (Color online) (a) The dependence of the correlation length $\xi_{x}$ against temperature $T$, calculated for noninteracting (dashed line) and dipole-dipole interacting (solid line) indirect excitons. (b) The $k_{\|}$-filtering effect: $\widetilde{g}^{(1)}=\widetilde{g}^{(1)}\left(r_{\|}\right)$evaluated for $\alpha$ $=18.9^{\circ}($ solid line $), 8.3^{\circ}$ (dashed line), $2.1^{\circ}$ (dotted line), $1.4^{\circ}$ (dashdotted line), and $0.8^{\circ}$ (dash-double-dotted line). Inset: the real-space 2D image of $\widetilde{g}^{(1)}$. (c) The coherence length $\xi_{\gamma}$ against the aperture angle $2 \alpha$.

$T_{0}$, i.e., when classical or weakly developed BE statistics are realized, and to a quantum gas of indirect excitons at $T$ $\leq T_{0}$, but still above the phase transition temperature. In all these cases the correlation function for a quasi-2D system of weakly interacting excitons is universally given by Eq. (6).

The optical spatial coherence function $\widetilde{g}^{(1)}$ of indirect excitons. In order to explain the experiments, ${ }^{4-7}$ which demonstrate a coherence length $\xi_{\gamma}$ much larger than $\xi_{x} \sim 0.1 \mu \mathrm{m}$, we implement the concept of $k_{\|}$-filtering. In this case, $\widetilde{g}^{(1)}$ $=\widetilde{g}_{\text {ind }}^{(1)}\left(r_{\|}\right)$is given by Eq. (3) with the efficiency of the "indirect exciton $\rightarrow$ bulk photon" conversion $\Gamma_{x-\gamma}=\left(2 k_{0}^{2}\right.$ $\left.-k_{\|}^{2}\right) /\left[k_{0}\left(k_{0}^{2}-k_{\|}^{2}\right)^{1 / 2}\right] .{ }^{8-10,19}$ In Fig. 3(b), we plot $\widetilde{g}_{\text {ind }}^{(1)}$ calculated for various aperture angles, $2^{\circ} \leqq 2 \alpha \lesssim 40^{\circ}$. The dependence $\widetilde{g}^{(1)}=\widetilde{g}_{\text {ind }}^{(1)}\left(r_{\|}\right)$is well approximated by Eq. (4). The above approximation of $\widetilde{g}^{(1)}$ by the "device function" $\widetilde{g}_{f}^{(1)}$ is valid when $n_{k_{\|}}=n_{E=\hbar^{2} k_{\|}^{2} / 2 M_{x}}$ is nearly constant in the rather narrow energy interval $0 \leq E \leq E^{(\alpha)}$, i.e., when $E^{(\alpha)}$ $=\left(\hbar k_{\|}^{(\alpha)}\right)^{2} / 2 M_{x} \ll k_{B} T e^{-T_{0} / T}$. For indirect excitons, this inequality with $T_{0}$ replaced by $T_{0}^{*}$ is definitely held for $n_{2 d}$ $\sim 10^{10} \mathrm{~cm}^{-2}$ and $T \sim 1 \mathrm{~K}$ (e.g., for $\alpha=20^{\circ}$ the cutoff energy $E^{(\alpha)}$ is only $\left.1.2 \mu \mathrm{eV}\right)$. Thus the $k_{\|}$-filtering effect yields the correlation length $\xi_{\gamma} \simeq 2.215 \sqrt{\varepsilon_{b}} /\left(k_{0} \sin \alpha\right)$, with $k_{0} \simeq 2.8$ $\times 10^{5} \mathrm{~cm}^{-1}$, according to Eq. (5). As a result, $\xi_{\gamma}$ is intrinsically scaled by the photon wavelength, i.e., is in the micron length scale [see Fig. 3(c), where $\xi_{\gamma}$ is plotted against the angle $\alpha$ ]. 
Compared to standard interference patterns in Young's double-slit experiment, with visibility contrast determined by $\widetilde{g}^{(1)}$, the oscillatory behavior of the optical coherence function $\widetilde{g}^{(1)}=\widetilde{g}^{(1)}\left(r_{\|}\right)$is rather unusual [see Eq. (4) and Fig. 3(b)]. This is a signature of the $k_{\|}$-filtering effect: The $k_{\|}$-filtering function $G_{f} \propto \Theta\left(k_{\|}^{(\alpha)}-k_{\|}\right)$gives a sharp cutoff at $k_{\|}=k_{\|}^{(\alpha)}$ in the integrals of Eq. (3) that results in oscillations of $\widetilde{g}^{(1)}\left(r_{\|}\right)$. In some aspects, the effect is similar to Friedel oscillations in a Fermi liquid, with $\hbar k_{\|}^{(\alpha)}$ akin to the Fermi momentum.

The coherence function $\widetilde{g}^{(1)}$ of MC polaritons. In this case, the "MC polariton $\rightarrow$ bulk photon" conversion function in Eq. (3) is $\Gamma_{x-\gamma}=\Psi\left(k_{\|}\right) / \tau_{\gamma}\left(k_{\|}\right)$, with $\Psi(0 \leq \Psi \leq 1)$ the photon component along a MC polariton branch and $\tau_{\gamma}$ the radiative (escape) lifetime of a MC photon. In Fig. $4, g^{(1)}=g_{\mathrm{MC}}^{(1)}\left(r_{\|}\right)$ calculated with Eq. (6) for circularly polarized MC polaritons is compared with $\widetilde{g}^{(1)}=\widetilde{g}_{\mathrm{MC}}^{(1)}\left(r_{\|}\right)$evaluated with Eq. (3). According to the experiments, ${ }^{2,3}$ we assume the BE distribution of MC polaritons along the lower polariton branch which is taken in the parabolic approximation with an effective in-plane mass $M_{\mathrm{MC}}^{l b}$. Compared to the case of QW excitons, the difference between $g_{\mathrm{MC}}^{(1)}$ and $\widetilde{g}_{\mathrm{MC}}^{(1)}$ is much smaller, still giving $\xi_{\gamma}>\xi_{p}$. This is because the cutoff energy $E^{(\alpha)}$ in the $k_{\|}$-filtering effect is much larger than that relevant to QW excitons, due to $M_{\mathrm{MC}}^{l b} \ll M_{x}$. If $k_{B} T \ll E^{(\alpha)} \sim 1 \mathrm{meV}, g_{\mathrm{MC}}^{(1)}$ and $\widetilde{g}_{\mathrm{MC}}^{(1)}$ nearly coincide (see Fig. 4 ).

We qualitatively explain a sharp increase in the coherence length with decreasing temperature, found in the experiments with coupled QWs, ${ }^{4-7}$ by combining the $k_{\|}$-filtering effect with screening of disorder by dipole-dipole interacting indirect excitons. ${ }^{17}$ In high-quality GaAs coupled QWs the screening process effectively develops at $T \lesssim 5 \mathrm{~K}$, giving

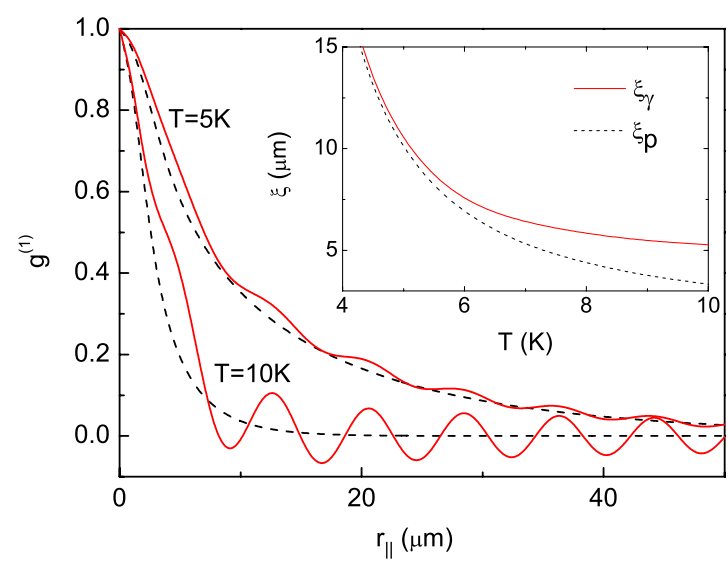

FIG. 4. (Color online) The MC polariton coherence function $g^{(1)}=g_{\mathrm{MC}}^{(1)}\left(r_{\|}\right)$(dashed lines) against that of emitted photons, $\widetilde{g}^{(1)}$ $=\widetilde{g}_{\mathrm{MC}}^{(1)}\left(r_{\|}\right)$(solid lines). Inset: the coherence lengths $\xi_{p}$ and $\xi_{\gamma}$ versus temperature $T$. The calculations, which model the experiments (Ref. 3) refer to a GaAs microcavity with positive detuning $\delta=7 \mathrm{meV}$ and Rabi splitting $\Omega_{\mathrm{MC}}=4 \mathrm{meV}$. The density of $\mathrm{MC}$ polaritons $n_{2 d}=10^{8} \mathrm{~cm}^{-2}$ and the aperture half-angle $\alpha=16.7^{\circ}$, so that $T_{0}$ $=27.6 \mathrm{~K}$ and $E^{(\alpha)}=0.96 \mathrm{meV}$.

rise to a well-defined single-particle momentum $\hbar \mathbf{k}_{\|}$, as has been observed, e.g., in the experiments. ${ }^{20,21}$ Thus the large correlation length $\xi=\xi_{\gamma} \sim 1 \mu \mathrm{m}$ can naturally be explained by the $k_{\|}$-filtering effect and cannot be interpreted as a signature of $\mathrm{BE}$ condensation in a system of indirect excitons.

We appreciate valuable discussions with L. V. Butov.
${ }^{1}$ I. Bloch, T. W. Hänsch, and T. Esslinger, Nature (London) 403 , 166 (2000).

${ }^{2}$ J. Kasprzak, M. Richard, S. Kundermann, A. Baas, P. Jeambrun, J. M. J. Keeling, F. M. Marchetti, M. H. Szymańska, R. André, J. L. Staehli, V. Savona, P. B. Littlewood, B. Deveaud, and Le Si Dang, Nature (London) 443, 409 (2006).

${ }^{3}$ H. Deng, G. S. Solomon, R. Hey, K. H. Ploog, and Y. Yamamoto, Phys. Rev. Lett. 99, 126403 (2007).

${ }^{4}$ S. Yang, A. T. Hammack, M. M. Fogler, L. V. Butov, and A. C. Gossard, Phys. Rev. Lett. 97, 187402 (2006).

${ }^{5}$ A. V. Gorbunov and V. B. Timofeev, Pis'ma Zh. Eksp. Teor. Fiz. 84, 390 (2006) [JETP Lett. 84, 329 (2006)].

${ }^{6}$ L. V. Butov, J. Phys.: Condens. Matter 19, 295202 (2007).

${ }^{7}$ V. B. Timofeev, in Problems of Condensed Matter Physics, edited by A. L. Ivanov and S. G. Tikhodeev (Oxford University Press, Oxford, 2008), pp. 258-284.

${ }^{8}$ E. Hanamura, Phys. Rev. B 38, 1228 (1988).

${ }^{9}$ L. C. Andreani, F. Tassone, and F. Bassani, Solid State Commun. 77, 641 (1991).

${ }^{10}$ D. S. Citrin, Phys. Rev. B 47, 3832 (1993).

${ }^{11}$ C. Klingshirn, Semiconductor Optics, 3rd ed. (Springer, Berlin, 2007), Sec. 23.4.
${ }^{12}$ R. J. Glauber, in Quantum Optics and Electronics, edited by C. DeWitt, A. Blandin, and C. Cohen-Tannoudji (Gordon and Breach, New York, 1965).

${ }^{13}$ M. O. Scully and M. S. Zubairy, Quantum Optics (Cambridge University Press, Cambridge, 1997).

${ }^{14}$ M. Naraschewski and R. J. Glauber, Phys. Rev. A 59, 4595 (1999).

${ }^{15}$ D. M. Kachintsev and S. E. Ulloa, Phys. Rev. B 50, 8715 (1994).

${ }^{16}$ L. V. Butov, J. Phys.: Condens. Matter 16, R1577 (2004).

${ }^{17}$ A. L. Ivanov, Europhys. Lett. 59, 586 (2002).

${ }^{18}$ A. L. Fetter and J. D. Walecka, Quantum Theory of ManyParticle Systems (McGraw-Hill, New York, 1971).

${ }^{19}$ A. L. Ivanov, P. B. Littlewood, and H. Haug, Phys. Rev. B 59, 5032 (1999).

${ }^{20}$ A. Parlangeli, P. C. M. Christianen, J. C. Maan, I. V. Tokatly, C. B. Soerensen, and P. E. Lindelof, Phys. Rev. B 62, 15323 (2000).

${ }^{21}$ L. V. Butov, A. L. Ivanov, A. Imamoglu, P. B. Littlewood, A. A. Shashkin, V. T. Dolgopolov, K. L. Campman, and A. C. Gossard, Phys. Rev. Lett. 86, 5608 (2001). 